\title{
Low-dose irradiation promotes proliferation of the human breast cancer MDA-MB-231 cells through accumulation of mutant P53
}

\author{
SI-JIE LI ${ }^{1}$, XIN-YUE LIANG ${ }^{2}$, HAI-JUN LI ${ }^{3}$, WEI LI ${ }^{2}$, LEI ZHOU ${ }^{2}$, \\ HUA-QIU CHEN ${ }^{4}$, SONG-GEN YE ${ }^{4}$, DE-HAI YU ${ }^{2}$ and JIU-WEI CUI ${ }^{2}$
}

${ }^{1}$ Department of Breast Surgery, ${ }^{2}$ Cancer Center, and ${ }^{3}$ Institute of Translational Medicine, The First Hospital of Jilin University, Changchun, Jilin 130021; ${ }^{4}$ Department of Clinical Medicine, Norman Bethane Health Science Center, Jilin University, Changchun, Jilin 130021, P.R. China

Received October 17, 2016; Accepted November 29, 2016

DOI: 10.3892/ijo.2016.3795

\begin{abstract}
Low-dose irradiation (LDIR) has been proven to have differential biological effects on normal mammalian somatic cells and cancer cells. Our previous study showed that $p 53$ gene status is a critical factor regulating the effect of LDIR on cancer cells. We investigated the effect of LDIR on the breast cancer cell line MDA-MB-231 that harbors a mutant p53 gene, and the normal breast fibroblast cell line Hs 578Bst. In the present study, we showed that $150 \mathrm{mGy}$ LDIR pormoted growth of MDA-MB-231 cells but not Hs 578Bst cells. Through cell cycle analyses, we found that LDIR accelerated cell cycle into $\mathrm{S}$ phase in MDA-MB-231 cells, but did not affect the cell cycle of Hs 578Bst cells. Using western blotting, we demonstrated that the expression of CDK4, CDK6 and cyclin D1 was upregulated in MDA-MB-231 cells after LDIR. Although LDIR increased ataxia-telangiectasia mutated (ATM) level in both MDA-MB-231 cells and Hs 578Bst cells and activated ATM/p53/p21 pathway, only the mutant type of P53 (mtP53) protein in MDA-MB-231 cells was shown to be accumulated after LDIR. Using ATM inhibitor or lentivirus-mediated small interfering RNA (siRNA) to block the ATM/p53/p21 pathway in MDA-MB-231 cells, the LDIR-induced cell proliferation was abolished. When we introduced wild-type P53 (wtP53) protein into MDA-MB-231 cells, the LDIR-induced cell proliferation was also abolished. These findings suggest that normal p53 function is crucial in ATM/p53/p21 pathway activated by LDIR. The p53 status is the most probable reason leading to
\end{abstract}

Correspondence to: Dr De-Hai Yu or Dr Jiu-Wei Cui, Cancer Center, The First Hospital of Jilin University, No. 71 Xinmin Street, Changchun, Jilin 130021, P.R. China

E-mail: yudehai@jlu.edu.cn

E-mail:cuijw@jlu.edu.cn

Key words: low-dose ionizing radiation, ataxia-telangiectasia mutated, p21, p53, breast cancer differential LDIR biological activities between breast tumor cells and normal breast cells.

\section{Introduction}

Breast cancer is the most common carcinoma among Chinese women. It is estimated that there will be an increasing number of new breast cancer patients in China (1). Radiation therapy may have a transformative impact on the treatment of breast cancer, because it allows women with early stage disease to maintain integrity of their body and those with advanced disease to have relief from suffering (2). Fisher et al (3) proved that breast-conserving surgery with radiation has the same therapeutic effect as mastectomy after their 20-year radomized trial in 1851 women with invasive breast tumors.

Although radiation therapy is a powerful anticancer modality, radiation-induced stress response and gene expression with an adaptive resistance may severely compromise the effectiveness of radiation (4). Mechanisms leading to radioresistance are diverse and still poorly defined. Many investigations have indicated that breast cancer stem cells evolve resistance to radiation due to intrinsic and extrinsic mechanisms, genetic mutations and epigenetic modifications (5).

Low-dose ionizing radiation (LDIR) is proved having hormesis $(6,7)$ and adoptive effect $(8)$, which is quite different from high-dose ionizing radiation (HDIR). Many investigations, incuding ours, have indicated that LDIR stimulates the proliferation of normal cells, such as rat mesenchymal stem cells, mouse bone marrow hematopoietic progenitor cells and several human normal cell lines $(9,10)$, but LDIR does not induce proliferation of tumor cells (11). Therefore, LDIR has been considering as a promising assistant method of clinical radiotherapy.

p53 plays a key role in the process of radiation response, controlling the activation of DNA repair and cell apoptosis pathways after acute radiation injury $(12,13)$. Since more than half of all tumors harbor $p 53$ gene mutation and deletion (14), it is necessary to investigate the biological behavior of these cells with abnormal p53 gene after LDIR. Our previous study demonstrated that $75 \mathrm{mGy}$ LDIR inhibits the proliferation of 
$p 53^{\text {null }}$ type human prostate tumor cells. In this investigation, we further illustrate how breast cancer cells with $p 53$ mutation responded to LDIR. We hope that the present study provides a valuable theoretical reference and supplement to the radiation treatment of breast cancer.

\section{Materials and methods}

Cell cultivation and treatments. The human breast cancer cell line MDA-MB-231 and normal breast fibroblast cell line Hs 578Bst were purchased from the American Type Culture Collection (ATCC; Manassas, VA, USA); human embryonic kidney cell line 293T was kept in our laboratory. MDA-MB-231 and 293T cells was maintained in Dulbecco's modified Eagle's media (DMEM; Life Technology, Shanghai, China) supplemented with $10 \%$ fetal bovine serum (FBS; Hyclone Laboratories, Beijing, China) and 1\% antibiotics (penicillin/streptomycin; Invitrogen, Carlsbad, CA, USA). Hs 578Bst cells were maintained in DMEM/F12 supplemented with $10 \% \mathrm{FBS}$ and $30 \mathrm{ng} / \mathrm{ml}$ epidermal growth factor (EGF; Invitrogen). The cells were cultured at $37^{\circ} \mathrm{C}$ in a humidified incubator with a constant air flow of $5 \% \mathrm{CO}_{2}$.

In order to inhibit the function of ATM, $10 \mu \mathrm{M}$ KU55933 (Selleck Chemicals, Co., Ltd., Shanghai, China) dissolved in dimethyl sulfoxide (DMSO; Sigma-Aldrich, Shanghai, China) was added to the cell culture media $2 \mathrm{~h}$ prior to irradiation. Media containing the chemical inhibitors were replaced with fresh medium immediately after the irradiation.

Lentiviral expression of wtP53 and p53 siRNA. The full-length $1.2 \mathrm{~kb}$ human p53 gene coding sequence (CDS) was amplified from the P53-expressing plasmid of pcDNA3.1(-)-P53 kept in our laboratory. The PCR primers contain the $\mathrm{XbaI}$ and EcoRV restriction sites. The primers sequence are as follows. Forward, 5'-ATAGAAGATTCTAGATGTACAGCCGCCACCATGGA GGAGCCGCAGTCAGAT-3' and reverse, 5'-TCAGTCTGAG TCAGGCCCTTTAATGAGATATCGTCGACGAAT-3'. The PCR product was gel-purified, cut by restriction enzymes and ligated into the $\mathrm{pCMV}$-copGFP/Puro vector constructed in our laboratory. The sense sequence of p53-siRNA and scrambled siRNA were CACCATCCACTACAACTACAT (15) and GGATTTCGAGTCGTCTTAA. The siRNA fragment was synthesized by PCR and cloned into pGreenPuro vector (SBI, Los Angeles, CA, USA) via restriction sites of BamHI and EcoRI. Both p53 overexpression and shRNA clones were confirmed by sequencing and then packaged in 293T packing cells using the method described in our laboratory (16). At $48 \mathrm{~h}$ post-transduction, cell clones were selected by $2 \mu \mathrm{g} / \mathrm{ml}$ puromycin (Invivogen, Inc., San Diego, CA, USA). After 7 days of puromycin-selection, stable transduction cells were used for LDIR.

Irradiation strategy. Monolayer cells were ionizing irradiated at the dose of $12.5 \mathrm{mGy} / \mathrm{min}$ by X-RAD 320 (Precision X-RAD; Precision X-Ray, North Branford, CT, USA). The total dose was 50, 100, 150 or 200 mGy. After LDIR, culture media were replaced and cells were harvested immediately or continually cultured until the next step of the experiment was carried out. Control groups were treated similarly except for irradiation.
Cell proliferation assay. Approximately $3 \times 10^{3}$ MDA-MB-231 cells and Hs 578Bst cells were seeded in a 96-well plate $24 \mathrm{~h}$ before LDIR, and then irradiated with $50,100,150$ or $200 \mathrm{mGy}$ of X-rays. After the LDIR, cells were transferred to the incubator and cultured for another 24,48 or $72 \mathrm{~h}$. Cell proliferation assays were determined by WST-1 cell proliferation reagent (Roche Diagnostics, Shanghai, China). According to the manufacturer's instructions, $20 \mu \mathrm{l}$ WST-1 reagent was added to $200 \mu \mathrm{l}$ cell culture medium and incubated in the dark for $2 \mathrm{~h}$. Then, the absorbance of $450 \mathrm{~nm}$ and $630 \mathrm{~nm}$ were measured by microplate reader (Bioteck, Co., Ltd., Beijing, China). Final OD (optical density) was designated as OD $4_{50}-\mathrm{OD}_{630^{-}}$ $\mathrm{OD}_{\text {blank }}$.

Cell cycle assay. Approximately $2 \times 10^{6}$ irradiated MDA-MB-231 cells or Hs 578Bst cells were harvested and washed by $1 \mathrm{ml}$ cold phosphate-buffered saline (PBS; Thermo Fisher Scientific, Beijing, China) twice to remove the residual trypsin and serum. Cells were resuspended in $1 \mathrm{ml}$ fixation solution (300 $\mu \mathrm{l}$ PBS and $700 \mu \mathrm{l}$ ethanol). After incubated at $4^{\circ} \mathrm{C}$ for $4 \mathrm{~h}$, cells were centrifuged at $1000 \mathrm{rpm}$ for $5 \mathrm{~min}$ and fixation solution was removed. After washed twice with $1 \mathrm{ml}$ PBS, cells were pelleted and suspended in $0.5 \mathrm{ml}$ propidium iodide (PI; Sigma-Aldrich) staining solution [50 $\mu \mathrm{g} / \mathrm{ml} \mathrm{PI,}$ $20 \mu \mathrm{g} / \mathrm{ml}$ RNase A (Takara Bio, Dalian, China) and $0.2 \%$ Triton X-100 (Sigma-Aldrich)] and incubated in the dark at $37^{\circ} \mathrm{C}$ for $30 \mathrm{~min}$. Cell suspensions were filtered through a 400-mesh sieve before analyzed by a BD FACSCalibur flow cytometer (Becton-Dickinson, Sparks, MD, USA).

Protein extraction and western blot analysis. Cell total protein was extracted with RIPA buffer (Beyotime Institute of Biotechnology, Shanghai, China) supplemented with cocktail protease inhibitor (Roche), and the protein concentration was determined by BCA protein assay kit (Beyotime Institute of Biotechnology).

Approximately 5-40 mg total protein was separated by 4-8\% (for ATM) or 5-12\% (for other proteins) SDS polyacrylamide gel electrophoresis (SDS-PAGE), and then was electrophoretically transferred to PVDF membranes $(0.45 \mu \mathrm{m}$; Millipore, Billerica, MA, USA) and blocked at $37^{\circ} \mathrm{C}$ for $1 \mathrm{~h}$ with $5 \%$ skim milk in TBST [TBS $(10 \mathrm{mmol} / 1$ Tris $\mathrm{pH} 7.5,150 \mathrm{mmol} / \mathrm{l} \mathrm{NaCl})$ containing 0.1\% Tween-20 (Sigma-Aldrich)]. Afterwards, membranes were incubated in monoclonal antibodies against CDK4 (1:1,500; Cell Signaling Technology, Beijing, China), CDK6 (1:1,500; Santa Cruz Biotechnology, Santa Cruz, CA, USA), cyclin D1 (1:1,500; Abcam, Shanghai, China), P53 (1:1,500; DO7, Santa Cruz Biotechnology), p21 (1:1,000, C-19; Santa Cruz Biotechnology), ATM (1:1,500, Y170; Cell Signaling Technology) and $\beta$-actin (1:3,000; Santa Cruz Biotechnology) at $4^{\circ} \mathrm{C}$ overnight. After washed for $3 \times 5 \mathrm{~min}$ with TBST, membranes were incubated with HRP conjugated goat anti-mouse or goat anti-rabbit second antibodies (1:3,000; ZSGB-BIO, Beijing, China) at $37^{\circ} \mathrm{C}$ for $1 \mathrm{~h}$. Washed further 3 times, the immunocomplexes were detected with the enhanced chemiluminescence system (ECL; Thermo Fisher Scientific) and X-ray film (Kodak, Beijing, China). Protein expression levels were determined semi-quantitatively by densitometric analysis with Quantity One software (Bio-Rad Laboratories). 
Statistical analysis. All data and results were calculated from at least three replicate measurements and were presented as means \pm SD. The significance was determined by the Student's $\mathrm{t}$-test using the SPSS 20.0 (IBM). $\mathrm{P}<0.05$ was considered statistically significant $\left({ }^{*} \mathrm{P}<0.05 ;{ }^{* *} \mathrm{P}<0.01 ; \mathrm{NS}\right.$, not significant).

\section{Results}

LDIR induces differential cell growth in breast cancer MDA-MB-231 and normal Hs 578Bst cells. In order to investigate the function of LDIR on breast cancer MDA-MB-231 and normal Hs 578Bst cells, we set up four different doses of LDIR including 50, 100, 150 and $200 \mathrm{mGy}$. Cell proliferation was assayed by WST-1 $24 \mathrm{~h}$ post-LDIR. Compared to the shamirradiated group, we found that 100 and $150 \mathrm{mGy}$ doses of LDIR significantly promoted the growth of MDA-MB-231 cells (Fig. 1A-b; P<0.01), and that the $150 \mathrm{mGy}$ irradiation had the most significant promotion effect. In contrast, none of the irradiation doses affected the growth of Hs 578Bst cells (Fig. 1A-a).

We compared the cell growth in two cell lines at different time-points following $150 \mathrm{mGy}$ LDIR. Cell proliferation was determined by WST- 1 at $0,24,48$ and $72 \mathrm{~h}$ post-LDIR. Apparently, MDA-MB-231 cells showed an accelerated proliferation $24 \mathrm{~h}$ post-LDIR (Fig. 1B-b; $<<0.01$ ). However, normal breast Hs 578Bst cells showed no obvious change after $150 \mathrm{mGy}$ irradiation compared with the sham control group (Fig. 1B-a; P>0.05). Cell morphology also showed the differential speed of cell growth between MDA-MB-231 cells and Hs 578Bst cells after LDIR (Fig. 1C).

LDIR accelerates entry into $S$ phase in MDA-MB-231 cells. We then used flow cytometry to analyze cell cycle distribution at $24 \mathrm{~h}$ post-LDIR. We found that LDIR may increase $S$ phase cell percentage in MDA-MB-231 cells compared to the sham group (Fig. 2A-c and -d). In contrast, we did not observe any significant change of cell cycle distribution in Hs 578Bst cells after LDIR (Fig. 2A-a and -b). Quantitation data showed that in MDA-MB-231 cells, the $S$ phase percentage was increased to 1.5 -fold (32.4 vs. $21.6 \%$, P $<0.01$; Fig. $2 \mathrm{~B}$ ).

Activation of cyclin dependent kinases. We further detected the expression of G1/S-phase related cyclins and cyclin-dependent kinases (CDKs). The expression of cyclin D1, cyclin E, CDK2, CDK4 and CDK6 was detected by western blotting at $24 \mathrm{~h}$ post-150 mGy LDIR. We observed a significant increment in cyclin-dependent kinases following LDIR (Fig. 2C-a). Quantitation analyses showed that the expression of CDK4, CDK6 and cyclin D1 significantly increased by 5.1-, 5.3- and 5.1-fold, respectively (Fig. 2C-b; $\mathrm{P}<0.01$ ). However, there was no significant changes in cyclin $\mathrm{E}$ and $\mathrm{CDK} 2$ following irradiation (data not shown).

LDIR specifically activates the ATM/p53/p21 pathway in breast cancer cells. We have demonstrated that ATM/p53/p21 pathway is a critical LDIR-associated pathway leading to cell cycle and cell proliferation change especially in $p 53$ abnormal cancer cells (unpublished data). We first checked the ATM level at $4 \mathrm{~h}$ post-LDIR. We found that the expression of ATM in both Hs 578Bst and MDA-MB-231 cells was upregulated by $150 \mathrm{mGy}$ LDIR at $4 \mathrm{~h}$ post-LDIR. In Hs 578Bst cells, the expres-
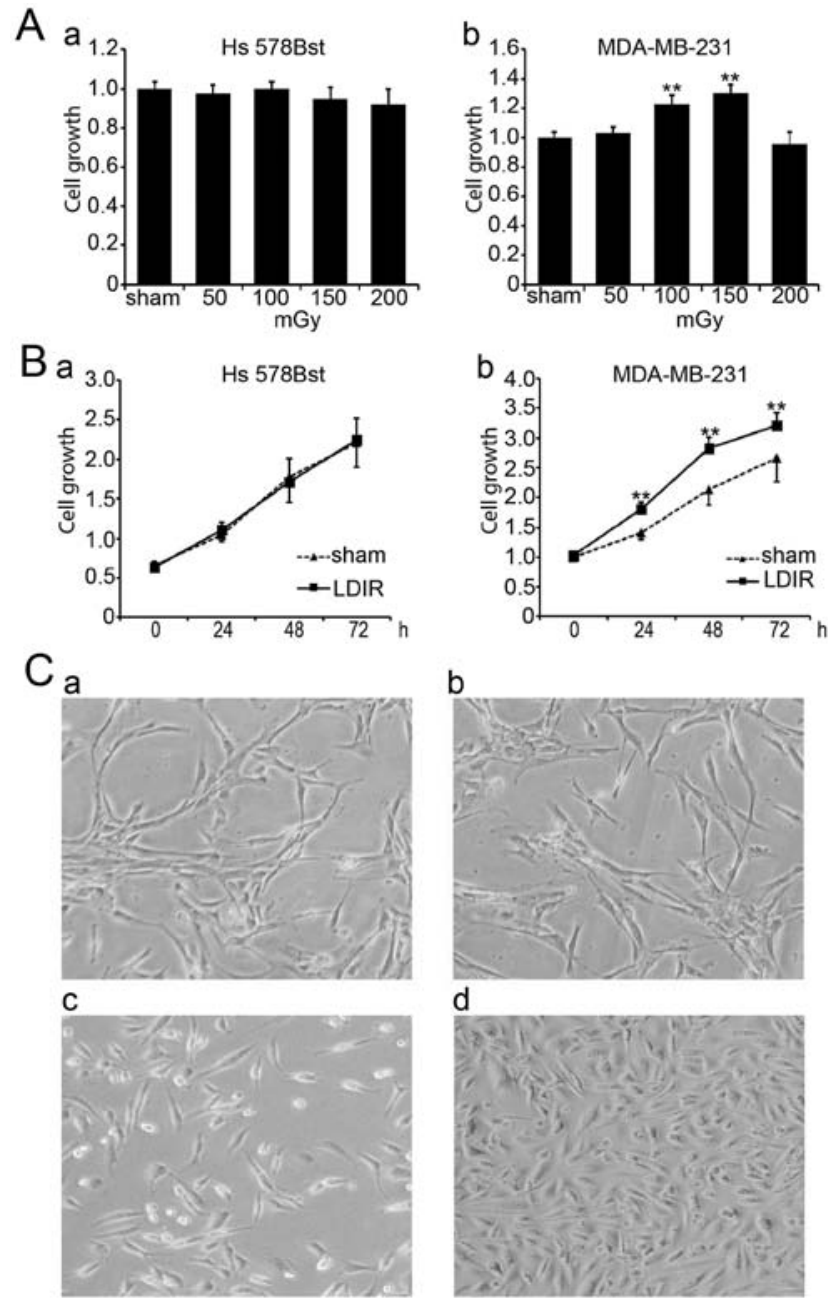

Figure 1. Low dose of irradiation induces differential response in (a) Hs 578Bst and (b) MDA-MB-231 cells. (A) Cell growth. Cells were irradiated by 50 , 100,150 and 200 mGy LDIR, and cell growth were determined by WST-1 at $24 \mathrm{~h}$ post-LDIR. MDA-MB-231 cells showed increased growth after 100 and $150 \mathrm{mGy}$ LDIR, and $150 \mathrm{mGy}$ had the most apparently stimulative effect; however, none of the four doses of LDIR affected the growth of normal breast Hs 578Bst cells. ${ }^{* *} \mathrm{P}<0.01$.(B) Growth situation of Hs 578Bst and MDA-MB-231 cells at 24,48 and $72 \mathrm{~h}$ post-LDIR. The growth situation of both Hs 578Bst and MDA-MB-231 cells after 150 mGy LDIR were recorded and cell proliferations at different time-points were determined by WST-1. (a) Hs 578Bst cells showed no change in proliferation after 150 mGy LDIR; (b) MDA-MB-231 cells showed significantly increased proliferation at 24,48 and $72 \mathrm{~h}$ post-LDIR. ${ }^{* *} \mathrm{P}<0.01$. (C) Cell morphology at $48 \mathrm{~h}$ post-irradiation. (a) Sham-irradiated Hs 578Bst cells; (b) 150 mGy irradiated Hs 578Bst cells. (c) Sham-irradiated MDA-MB-231; (d) 150 mGy irradiated MDA-MB-231 cells.

sion of ATM was upregulated by 3-fold and in MDA-MB-231 cells, it was activated by 2.8 -fold (Fig. $3 \mathrm{~A}$ and $\mathrm{B}$-a; $\mathrm{P}<0.01$ ). Then we examined the expression of P53. Western blot results revealed that the expression of P53 increased by 1.3 -fold at $4 \mathrm{~h}$ post-LDIR in MDA-MB-231 cells (Fig. 3A and B-b; $\mathrm{P}<0.05$ ). We also quantitated the expression of p21 after LDIR. In MDA-MB-231 cells, we observed a significant downregulation of p21 by 0.4 -fold after LDIR (Fig. 3A and B-c; P<0.01). However, the LDIR-induced P53 and p21 changes were absent in normal Hs 578Bst cells.

ATM inhibitor and P53 siRNA interferes LDIR-induced cell proliferation in MDA-MB-231 cells. To further address the role of the ATM/p53/p21 pathway in the LDIR-induced cell 

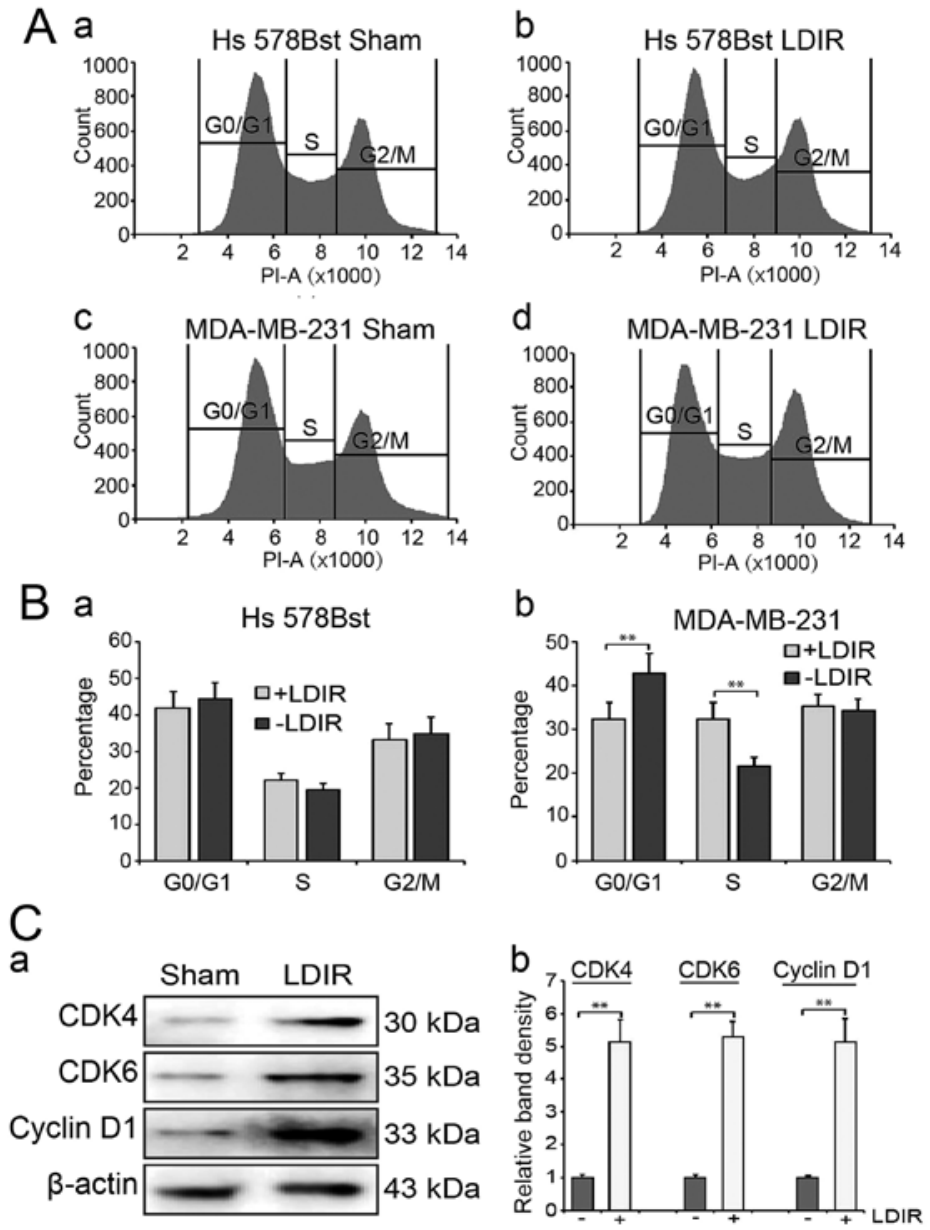

Figure 2. LDIR accelerates entry into S phase in MDA-MB-231 cells. (A) Cell cycle distribution of Hs 578Bst and MDA-MB-231 cells. Following LDIR, cells were determined by flow cytometry at $24 \mathrm{~h}$ post-LDIR. (a) Sham-irradiated Hs 578Bst cells; (b) 150 mGy irradiated Hs 578Bst cells. (c) Sham-irradiated MDA-MB-231; (d) 150 mGy irradiated MDA-MB-231 cells. (B) (a) (b) Average percentages of cells in each cell cycle phase. ${ }^{* *} \mathrm{P}<0.01$. (C) LDIR activated cyclin dependent kinases. (a) Western blotting; (b) relative band density. ${ }^{* *} \mathrm{P}<0.01$.
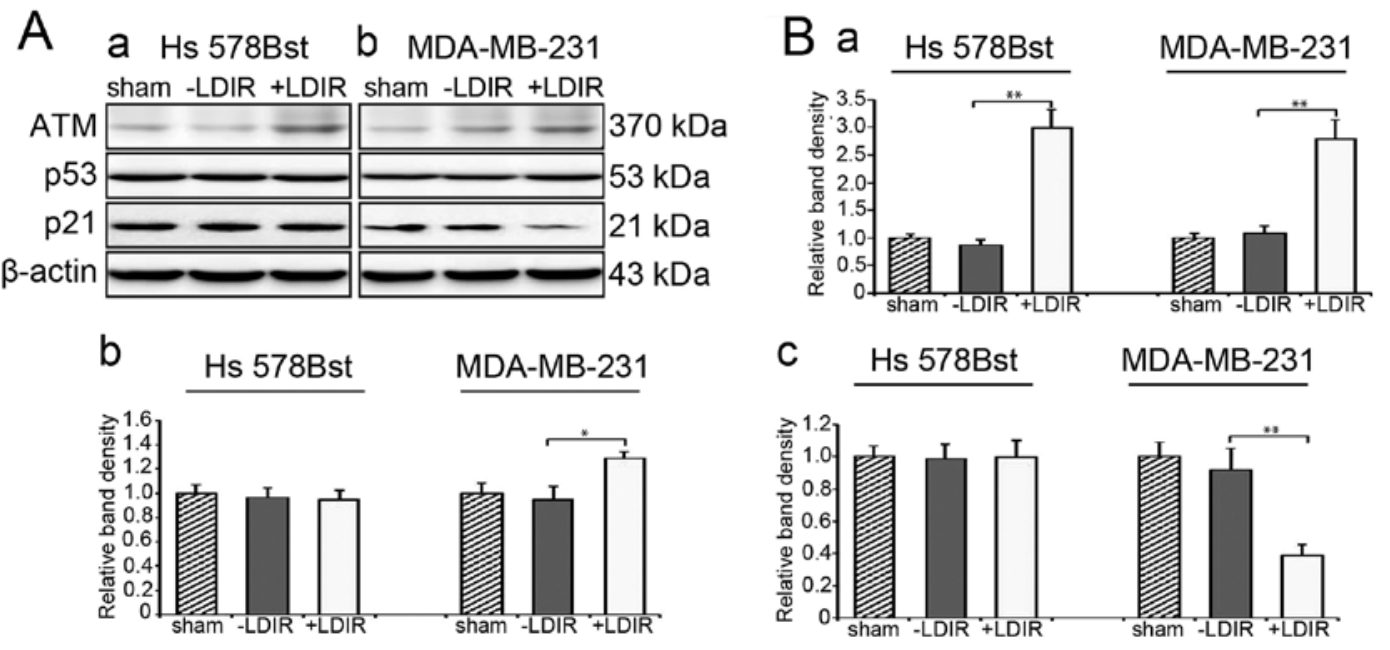

Figure 3. LDIR activates ATM/p53/p21 pathway in MDA-MB-231 cells. (A) Detection of ATM, p53 and p21 expression by western blotting. (B) Relative blotting band density of (a) ATM, (b) p53 and (c) p21. ${ }^{*} \mathrm{P}<0.05$; ${ }^{* *} \mathrm{P}<0.01$.

proliferation change, we used KU55933 to block the function of ATM in MDA-MB-231 cells and measured the cell proliferation at $24 \mathrm{~h}$ post-LDIR. We found that the LDIR induced proliferation in the MDA-MB-231 cells was abolished after the treatment of KU55933 (Fig. 4C-a; P<0.05). We also used lentiviral p53 shRNA to knock down the expression of mtP53 in the MDA-MB-231 cells (Fig. 4A), and the LDIR induced proliferation was abolished, (Fig. 4C-b; $\mathrm{P}<0.05$ ). 

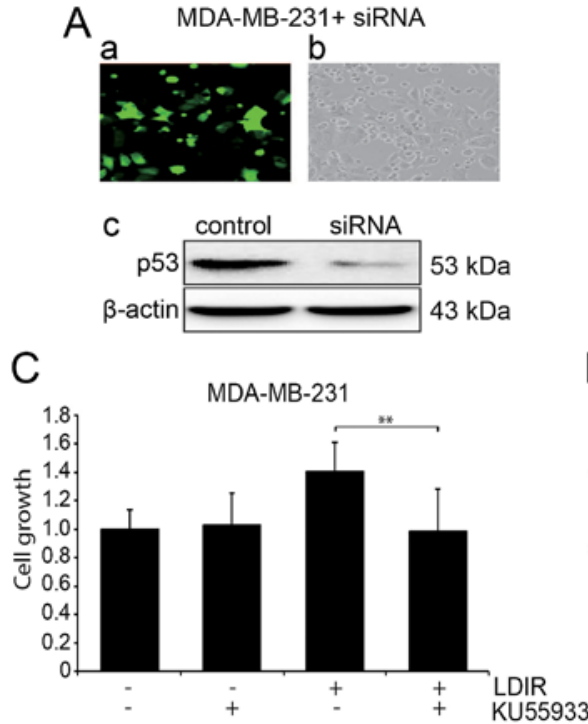
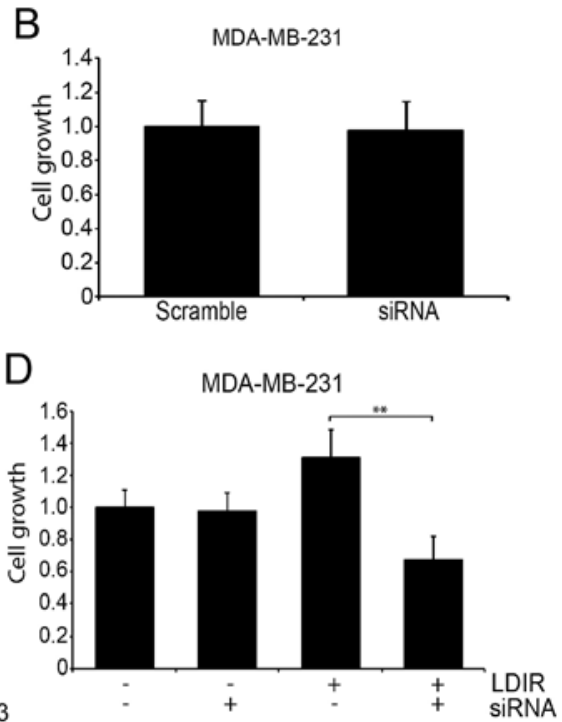

Figure 4. ATM inhibitor and P53 siRNA interferes LDIR-induced cell proliferation in MDA-MB-231 cells. (A) Construction of p53 knock down stable cell line. (a and b) MDA-MB-231 cells were transduced by GFP-tagged lentiviral shRNA. (c) Detection of p53 expression via western blotting. (B) There was no difference in cell proliferation between scramble shRNA group and p53 shRNA group. (C) ATM inhibitor KU55933 neutralized LDIR induced cell proliferation in MDA-MB-231 cells. Cells were treated by $10 \mu \mathrm{M}$ KU55933 $2 \mathrm{~h}$ prior to cell irradiation by $150 \mathrm{mGy}$ LDIR. Cell growth was determined by WST-1 at $24 \mathrm{~h}$ post-LDIR. (D) p53 siRNA neutralized LDIR induced cell proliferation in MDA-MB-231 cells. MDA-MB-231 cells that stably express lentiviral shRNA were irradiated by $150 \mathrm{mGy}$ LDIR. Cell growth was determined by WST-1 at $24 \mathrm{~h}$ post-LDIR.
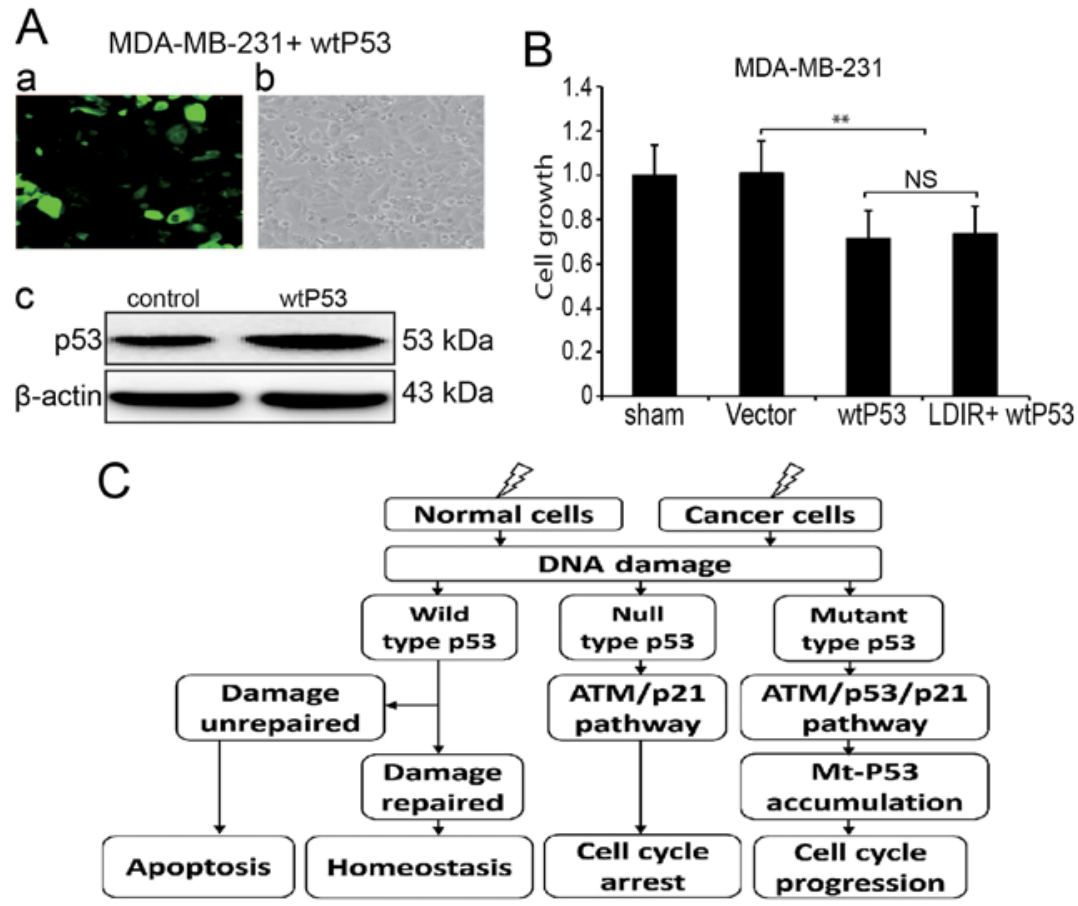

Figure 5. Wild-type P53 interferes LDIR-induced cell proliferation in MDA-MB-231 cells. (A) Construction of wtP53 expression stable cell line. (a and b) MDA-MB-231 cells were transduced by GFP-tagged lentiviral vector. (c) Detection of p53 expression via western blotting. (B) wtP53 neutralized LDIR induced cell proliferation in MDA-MB-231 cells. MDA-MB-231 cells that stably express wtP53 were irradiated by 150 mGy LDIR and cell growth was determined by WST-1 at $24 \mathrm{~h}$ post-LDIR. (C) The putative mechanism underlying the differential biological effect after LDIR between normal cells and $p 53$ dysfunctional cancer cells.

Wild-type P53 interferes LDIR-induced cell proliferation in MDA-MB-231 cells. Since MDA-MB-231 expresses mtP53, we introduced wtP53 by transducing MDA-MB-231 cells with lentivirus (Fig. 5A). We investigated the proliferation of wtP53 transduced MDA-MB-231 cells as well as cells irradiated by $150 \mathrm{mGy}$ LDIR. We found that the LDIR-induced cell proliferation was also abolished (Fig. 5B).

\section{Discussion}

The linear no-threshold (LNT) model assumes that even very low doses of ionizing radiation may generate detrimental effects on human health (17). However, it is indicated that although ionizing radiation can harm cells, such injurious effects are not linear with the radiation dose mainly due to 
different cellular mechanisms to adapt or die $(18,19)$. Besides, there is increasing evidence showing that radiation below certain doses could be protective $(20,21)$.

LDIR is usually defined as $\leq 0.2$ Gy at low linear energy transfer (LET) or $\leq 0.05$ Gy at high LET (22). In general, biological effects of LDIR can be summarized as 'hormesis', 'adaptive response (AR)', 'bystander effects', 'hyper-radiosensitivity (HRS)' and 'induced radioresistance (IRR)' (23). These biological effects of LDIR, especially hormesis and $\mathrm{AR}$, have been investigated for the potential applicability in the treatment of cancer due to its suppressive effect on cancer induction, growth and metastasis (24). However, the health risks associated with LDIR are still non-negligible, and owing to a lack of understanding of the mechanisms underlying the response of LDIR, the potential applicability of LDIR in clinical anticancer therapy remains controversial. Therefore, it is worthwhile to further clarify and provide more investigation evidence of LDIR on molecular level.

Our previous study elucidated that ATM/p53/p21 is an important responding pathway to LDIR in human prostate cells (unpublished data). Because of a lack of normal p53 function, the proliferation of PC-3 cells was suppressed by LDIR. In the present study, we chose MDA-MB-231 cells to investigate the biological effect of LDIR on breast cancer. MDA-MB-231 cells have a mutation on $p 53$ gene at exon 8 (25). We investigated the cell proliferation after 50-200 mGy LDIR. MDA-MB-231 cells showed its radiation sensitivity at 100 and 150 mGy LDIR at the dose rate of $12.5 \mathrm{mGy} / \mathrm{min}$, but the normal breast fibroblast cell line Hs 578Bst did not respond to LDIR. In our series of studies, we have investigated the LDIR biological effects on several cancer cell lines, such as K562, HL-60, NCI-H446, BEL7402, U251, HCT-8 and HeLa (9-11). However, we found LDIR has no obvious effect on these cancer cell lines. It is noteworthy that in these investigations, we did not focus on the genetic backgrounds of these cells, until we found that the proliferation of PC-3 cells were inhibited by LDIR.

When the cell cycle distribution was analyzed, we found LDIR accelerates the cell cycle entry into S phase in MDA-MB-231 cells, and the expression of CDK4, CDK6 and cyclin D1 increases. When we further investigated the molecular mechanism during this process, we found that ATM/p53/p21 pathway was also activated by LDIR in MDA-MB-231 cells. Although ATM in both MDA-MB-231 cells and Hs 578Bst cells was activated by LDIR, only the mtP53 protein in MDA-MB-231 cells was shown to be increased.

The $p 53$, as a tumor suppressor gene, is crucial in the protection against DNA damage and other forms of physiological stress primarily by inducing cell cycle arrest or apoptosis (26). Mutation of p53, however, inactivates these growth regulatory functions and causes a loss of tumor suppressor activity. It has been reported that overexpression of mtP53 proteins in $p 53^{\text {null }}$ cells resulted in enhancement of plating efficiency and tumorigenicity $(27,28)$. Stable expression of $\mathrm{p} 53$ plays a central role in LDIR activated ATM/p53/p21 pathway, affecting cell proliferation and cell cycle. In normal cells, radiation-induced DNA damage can be repaired by p53 and cells maintain homeostasis; in breast cancer MDA-MB-231 cells, our results indicated that the ATM/p53/p21 pathway was also activated by LDIR. However, since mtP53 has an increased half-life (29), the fast growth of MDA-MB-231 cells can be ascribed to the abnormal accumulation of mtP53 (Fig. 5C).

Of importance, different cells and organs may respond differently to the same kind of radiation even at the same dose level. Differential genetic backgrounds of cells and tumor microenvironment also influence the activity of signaling pathways. Therefore, further investigation directed toward the tumor niche should be carried out to elucidate the biological effect of LDIR.

\section{Acknowledgements}

The present study was supported by grants from the National Science Foundation of China (81302380, to D.H.Y), and the Jilin Provincial Science and Technology Department (20140520017JH).

\section{References}

1. GLOBOCAN: Estimated Cancer Incidence, Mortality and Prevalence Worldwide in 2012. http://globocan.iarc.fr/Default. aspx. Access date: June 10, 2016.

2. Rodin D, Knaul FM, Lui TY and Gospodarowicz M: Radiotherapy for breast cancer: The predictable consequences of an unmet need. Breast 29: 120-122, 2016.

3. Fisher B, Anderson S, Bryant J, Margolese RG, Deutsch M, Fisher ER, Jeong JH and Wolmark N: Twenty-year follow-up of a randomized trial comparing total mastectomy, lumpectomy, and lumpectomy plus irradiation for the treatment of invasive breast cancer. N Engl J Med 347: 1233-1241, 2002.

4. Kim JJ and Tannock IF: Repopulation of cancer cells during therapy: An important cause of treatment failure. Nat Rev Cancer 5: 516-525, 2005.

5. Peitzsch C, Kurth I, Kunz-Schughart L, Baumann M and Dubrovska A: Discovery of the cancer stem cell related determinants of radioresistance. Radiother Oncol 108: 378-387, 2013

6. Luckey TD: Physiological benefits from low levels of ionizing radiation. Health Phys 43: 771-789, 1982.

7. Feinendegen LE: Evidence for beneficial low level radiation effects and radiation hormesis. Br J Radiol 78: 3-7, 2005.

8. Olivieri G, Bodycote $\mathrm{J}$ and Wolff S: Adaptive response of human lymphocytes to low concentrations of radioactive thymidine. Science 223: 594-597, 1984.

9. Li W, Wang G, Cui J, Xue L and Cai L: Low-dose radiation (LDR) induces hematopoietic hormesis: LDR-induced mobilization of hematopoietic progenitor cells into peripheral blood circulation. Exp Hematol 32: 1088-1096, 2004.

10. Liang X, So YH, Cui J, Ma K, Xu X, Zhao Y, Cai L and Li W: The low-dose ionizing radiation stimulates cell proliferation via activation of the MAPK/ERK pathway in rat cultured mesenchymal stem cells. J Radiat Res (Tokyo) 52: 380-386, 2011.

11. Jiang H, Xu Y, Li W, Ma K, Cai L and Wang G: Low-dose radiation does not induce proliferation in tumor cells in vitro and in vivo. Radiat Res 170: 477-487, 2008.

12. Lee CL, Blum JM and Kirsch DG: Role of p53 in regulating tissue response to radiation by mechanisms independent of apoptosis. Transl Cancer Res 2: 412-421, 2013.

13. Menon V and Povirk L: Involvement of p53 in the repair of DNA double strand breaks: Multifaceted roles of p53 in homologous recombination repair (HRR) and non-homologous end joining (NHEJ). Subcell Biochem 85: 321-336, 2014.

14. Freed-Pastor WA and Prives C: Mutant p53: One name, many proteins. Genes Dev 26: 1268-1286, 2012.

15. Kim JS, Lee C, Bonifant CL, Ressom H and Waldman T: Activation of p53-dependent growth suppression in human cells by mutations in PTEN or PIK3CA. Mol Cell Biol 27: 662-677, 2007.

16. Zhang H, Zeitz MJ, Wang H, Niu B, Ge S, Li W, Cui J, Wang G, Qian G, Higgins MJ, et al: Long noncoding RNA-mediated intrachromosomal interactions promote imprinting at the Kcnq1 locus. J Cell Biol 204: 61-75, 2014.

17. Lindell B and Sowby D: The 1958 UNSCEAR report. J Radiol Prot 28: 277-282, 2008. 
18. Kadhim M, Salomaa S, Wright E, Hildebrandt G, Belyakov OV Prise KM and Little MP: Non-targeted effects of ionising radiation - implications for low dose risk. Mutat Res 752: 84-98, 2013.

19. Stankevicins L, Almeida da Silva AP, Ventura Dos Passos F, Dos Santos Ferreira E, Menks Ribeiro MC, G David M, J Pires E, Ferreira-Machado SC, Vassetzky Y, de Almeida CE, et al: MiR-34a is up-regulated in response to low dose, low energy X-ray induced DNA damage in breast cells. Radiat Oncol 8: 231, 2013.

20. Lehrer S and Rosenzweig KE: Lung cancer hormesis in high impact states where nuclear testing occurred. Clin Lung Cancer 16: 152-155, 2015

21. Dobrzynski L, Fornalski KW and Feinendegen LE: Cancer mortality among people living in areas with various levels of natural background radiation. Dose Response 13: $1559325815592391,2015$.

22. Mettler FA, Sinclair WK, Anspaugh L, Edington C, Harley JH, Ricks RC, Selby PB, Webster EW and Wyckoff HO: The 1986 and 1988 UNSCEAR (United Nations Scientific Committee on the Effects of Atomic Radiation) reports: Findings and implications. Health Phys 58: 241-250, 1990.
23. Yang G, Li W, Jiang H, Liang X, Zhao Y, Yu D, Zhou L, Wang G, Tian H, Han F, et al: Low-dose radiation may be a novel approach to enhance the effectiveness of cancer therapeutics. Int J Cancer 139: 2157-2168, 2016.

24. Liu SZ: On radiation hormesis expressed in the immune system. Crit Rev Toxicol 33: 431-441, 2003.

25. Bartek J, Iggo R, Gannon J and Lane DP: Genetic and immunochemical analysis of mutant p53 in human breast cancer cell lines. Oncogene 5: 893-899, 1990.

26. Cadwell $\mathrm{C}$ and Zambetti GP: The effects of wild-type p53 tumor suppressor activity and mutant p53 gain-of-function on cell growth. Gene 277: 15-30, 2001.

27. Shaulsky G, Goldfinger N and Rotter V: Alterations in tumor development in vivo mediated by expression of wild type or mutant p53 proteins. Cancer Res 51: 5232-5237, 1991.

28. Dittmer D, Pati S, Zambetti G, Chu S, Teresky AK, Moore M, Finlay C and Levine AJ: Gain of function mutations in p53. Nat Genet 4: 42-46, 1993.

29. Yan W, Zhang Y, Zhang J, Liu S, Cho SJ and Chen X: Mutant p53 protein is targeted by arsenic for degradation and plays a role in arsenic-mediated growth suppression. J Biol Chem 286: 17478-17486, 2011. 